Research Paper

\title{
Radiotherapy, Bisphosphonates and Surgical Stabilization of Complete or Impending Pathologic Fractures in Patients with Metastatic Bone Disease
}

\author{
Michal J. Wolanczyk ${ }^{\circledR}$, Khashayar Fakhrian², Irenäus A. Adamietz ${ }^{2}$ \\ 1. Department of General and Interventional Radiology and Neuroradiology, Wroclaw Medical University, Poland \\ 2. Department of Radiation Oncology, Ruhr-University of Bochum, Germany \\ $\square$ Corresponding author: Michal J. Wolanczyk, M.D., Department of General and Interventional Radiology and Neuroradiology, ul. Borowska 213, 50-556 \\ Wrocław, Poland. Tel.: 007173316 68, Fax: 00717341689 \\ ( ) Ivyspring International Publisher. Reproduction is permitted for personal, noncommercial use, provided that the article is in whole, unmodified, and properly cited. See \\ http://ivyspring.com/terms for terms and conditions.
}

Received: 2015.07.29; Accepted: 2015.11.01; Published: 2016.01.01

\begin{abstract}
Purpose: To report the treatment outcomes of patients with metastatic bone disease with complete or impending pathologic fractures, who were treated with postoperative radiotherapy (RT), bisphosphonates or both after orthopedic stabilization.

Material and Methods: We retrospectively evaluated the results of RT, bisphosphonates or both after orthopedic stabilization for complete or impending pathologic fractures in 72 patients with skeletal metastases. After surgery, 32 patients (44\%) were treated with RT alone (group 1), 31 patients (43\%) were treated with RT and bisphosphonates (group 2) and $9(13 \%)$ patients were treated with bisphosphonates (group 3), respectively. Patients were treated with a median dose of 30Gy (30-40 Gy/2-3Gy per fraction). The local tumor progression, pain progression and need for re-operation or re-radiotherapy were assessed from patients' medical records. Median follow-up time was 9 months.

Results: Median overall survival time was 14 months (95\% Cl: 12-17). Secondary surgical intervention at the same location was necessary in 1 patient of group $1(2 \%), 2$ patients of group $2(5 \%)$ and 2 patients of group $3(15 \%)$, respectively $(p=0.097)$. Local tumor progress was observed in 3 patients of group $1(9 \%), 2$ patients of group $2(7 \%)$ and 4 patients in group $3(44 \%)$, respectively $(p=0.021)$. Local pain progress was observed in $19 \%, 16 \%$ and $67 \%$ of the same groups $(p=0.011)$.

Conclusion: Our data confirm the efficacy and necessity of postoperative RT after orthopedic stabilization for metastatic bone disease to control the local disease. Bisphosphonates do not obviate the need for RT in the management of bone metastases after surgical stabilization. The combined treatment might lead to a better local tumor and pain control.
\end{abstract}

Key words: Pathological fracture, bisphosphonates, bone metastasis, orthopedic stabilization, postoperative radiotherapy

\section{Introduction}

External beam radiotherapy (EBRT) continues to be the mainstay for the treatment of pain and/or prevention of the morbidity caused by bone metastases. However, complete and impending pathologic fractures should be treated with durable fixation or reconstruction that will outlast the patient's expected survival (1). The major challenge for the orthopedic procedure is to achieve stability and decrease metastatic pain in the region of the lesion or fracture. In general, treatment of an impending pathologic fracture is less complicated than treatment of an actual fracture. Furthermore, elective fixation prevents the 
intense pain and loss of function associated with a pathologic fracture. Therefore, a goal of managing patients with bone metastases is prophylactic surgical fixation of identified bones that are at risk of developing a pathologic fracture before they actually fracture (2). Pathologic fractures resulting from metastatic disease are treated by repairing or removing existing bone (3). Intramedullary nailing or an implantation of a plate augmented with polymethylmethacrylate is the most common strategies. In the case of massive bone loss or a destroyed joint surface, the bone may be removed and replaced with a prosthesis (4). Unfortunately, tumor progression in the surgically supplied bone is common. Patients treated only with surgery present with poor outcomes of functional recovery. Indeed, within the first 5 months following surgical intervention, only about $30 \%$ of patients reach normal functional status (5). Postoperative percutaneous irradiation is necessary to eliminate residual microscopic disease and thus prevent disease progression and further osteolysis (6). By destroying tumor cells, radiotherapy (RT) achieves pain relief, reverses inflammation resulting from bone metastasis, and promotes the ossification of lytic lesions (7). Several studies have reported that the frequency of skeletal-related events such pain, pathologic fracture, hypercalcemia, and spinal cord compression can be reduced through use of osteoclast inhibitors such as bisphosphonates (8-10). Data reporting the results of postoperative treatment with RT, bisphosphonates or both after orthopedic stabilization in patients with metastatic bone disease are scarce. We evaluated the treatment outcomes of patients with metastatic bone disease with complete or impending pathologic fractures, who were treated with postoperative RT, bisphosphonates or both after orthopedic stabilization.

\section{Material and Methods}

Between January 2003 and December 2011, 72 patients with complete or impending pathologic fractures were treated with RT, bisphosphonates or both after orthopedic stabilization at the Ruhr-University of Bochum. The medical records, obtained from clinical charts of different departments involved in treatment (i.e., from admissions, surgical, internal, and radiation therapy departments and also from lab and imaging studies), were reviewed and used as a basis for evaluation of the endpoint. After surgery, 32 patients ( $44 \%$ ) were treated with RT alone (group 1), 31 patients (43\%) were treated with RT and bisphosphonates (group 2) and 9 (13\%) patients were treated with bisphosphonates (group 3), respectively. Patients were treated with a median dose of 30Gy (30-40 Gy/2-3Gy per fraction). The local tumor pro- gression, pain progression and need for re-operation or re-radiotherapy were assessed from patients' medical records. Table 1 shows baseline characteristics for all patients.

Table 1. Patients' baseline characteristics.

\begin{tabular}{ll}
\hline Characteristics & All patients, $\mathrm{n}=72(100 \%)$ \\
\hline Gender & $30(42 \%)$ \\
Male & $42(58 \%)$ \\
Female & $70(40-89)$ \\
Age (y), median (range) & $32(44 \%)$ \\
$\geq 70$ & $40(56 \%)$ \\
$\leq 70$ & \\
Primary cancer site & $23(32 \%)$ \\
Breast & $14(19 \%)$ \\
Colo-rectal & $11(15 \%)$ \\
Lung & $5(7 \%)$ \\
Prostate & $19(27)$ \\
Other & \\
Radiotherapy dose/fraction & $25(35 \%)$ \\
$30 / 10$ & $14(19 \%)$ \\
$40 / 2$ & $40(56 \%)$ \\
Other (between 30-40Gy/2-3Gy per fraction) \\
Osteolytic metastases & $54(75 \%)$ \\
Osteoblastic metastases & $18(25 \%)$ \\
Soft tissue infiltration & \\
Yes & $18(25 \%)$ \\
No & $54(75 \%)$ \\
Bone site & \\
Spine & $21(71 \%)$ \\
Long bones & $51(29 \%)$ \\
\hline
\end{tabular}

\section{Radiation therapy}

Postoperative EBRT was defined as radiation treatment that started within 8 weeks after surgical intervention (mean: 4 weeks). A decision to refer the patient for postoperative RT was made principally by an interdisciplinary board after the patient had been sent for a consultation by the treating orthopedic surgeon. Radiotherapy was performed using single beam in $19 \%$, two beams in $51 \%$, multiple isocentric beams in $8 \%$ and $3 \mathrm{D}$-conformal beams in $23 \%$ of patients. All patients were treated with a linear accelerator using a $6 \mathrm{MV}$ or $15 \mathrm{MV}$ photon beam. The total dose ranged from 30 to $40 \mathrm{~Gy}$ (median dose: $30 \mathrm{~Gy}$ ). The most frequently prescribed fractionation schemes was $10 \times 3$ Gy $(n=26)$.

\section{Bisphosphonates}

Forty patients received $4 \mathrm{mg}$ of Zoledronic acid each 3-4 week, starting 3 to 8 weeks after the surgical intervention.

\section{Follow-up}

During the first year after treatment was completed, patients were seen every 3 months. Skeletal-related events such pain, pathologic fracture, hy- 
percalcemia, and spinal cord compression were documented. Pain was evaluated with the help of a scale from 0 to 10, with boundaries of 0 representing no pain and 10 representing maximal pain. Pain flare was defined as a 2-point increase in the worst pain score (0-10) compared with baseline worst pain with no decrease in analgesic intake, or a $25 \%$ increase in analgesic intake with no decrease in worst pain score at the time of follow-ups. Diagnostic imaging was only performed in case of symptom exacerbation. Local progress was defined as any symptomatic increase in size of the tumor. Median follow-up time was 9 months for surviving patients (2 weeks-20 months).

\section{Statistics}

All time estimates began from the date of surgical stabilization. Overall survival (OS) was defined as death from any cause. All calculations were done with IBM SPSS 22 package software. Survival curves were compared by log-rank test. Baseline characteristics were compared by chi-square test. Fisher's exact tests were performed to compare distributions of categorical variables between independent groups. A two-sided level of significance of $\alpha=5 \%$ was considered to be statistically significant for all tests.

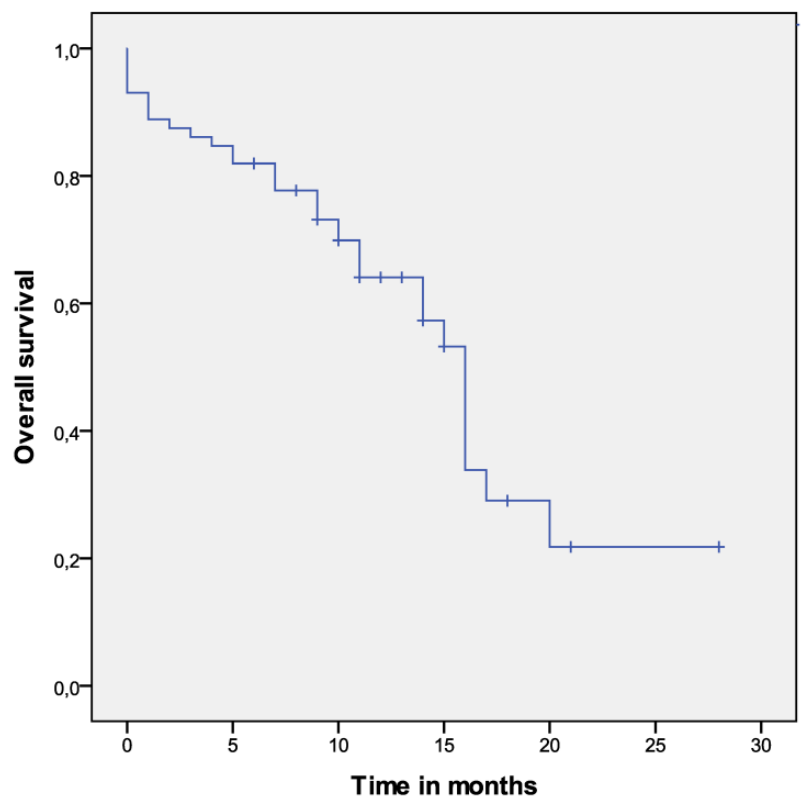

Figure 1. Overall survival of the whole cohort.

\section{Results}

Median overall survival time was 14 months (95\% CI: 12-17). Secondary surgical intervention at the same location was necessary in 1 patient of group $1(2 \%), 2$ patients of group $2(5 \%)$ and 2 patients of group $3(15 \%)$, respectively $(p=0.097)$. Local tumor progress within 6 months after surgery was observed in 3 patients of group $1(9 \%), 2$ patients of group 2 $(7 \%)$ and 4 patients in group $3(44 \%)$, respectively $(p=0.021)$. The overall pain flare incidence across all three groups was $20 / 72(28 \%)$. The incidence of pain flare was 6/32 (19\%) for group 1, 5/31 (16\%) for group 2 and $6 / 9(67 \%)$ for group 3 , respectively $(\mathrm{p}=0.011)$.

\section{Overall survival of the investigated group}

Twelve months after surgery, $65 \%$ of patients were still alive. The overall survival time for the whole group was 14 months (95\% CI: 12-17). Poor performance status $(p=0.001)$ and obviating the RT $(p=0.027)$ were significantly associated with shorter OS.

\section{Discussion}

Metastatic bone disease causes several complications related to deteriorating metabolic and mechanical function of the skeleton. Pathological bone fractures are feared complications of bone metastases because they mean a sudden, unexpected loss of function combined with a severe pain. The reason is an infiltration by tumor cells causing more or less extended destruction of bone. RT in the treatment of bone metastases is very effective (11-13). The local pain is sufficiently reduced, and long-lasting recalcification of bone may be achieved (13). However, surgical decompression and postoperative radiotherapy is recommended for spinal cord compression or instability of bones in highly selected patients with sufficient performance status and life expectancy (2). Unfortunately, this environment provides excellent conditions for malignant re-growth after surgery, resulting in a relapse and deterioration of function. RT can effectively eliminate the residual tumor cells in the bone marrow and in the vicinity of stabilizing material. Systemic anticancer therapies are frequently used for treatment of metastatic disease, but they do little independently to assist in the healing or treatment of pathologic fractures. Similarly, the use of bisphosphonates can prevent skeletal complications, such as fractures in patients with bone metastases, and may also improve pain related to bone metastases, but they are not adequate by themselves to aid healing of a pathologic fracture (2). Townsend et al. reviewed 64 orthopedic stabilization procedures in 60 consecutive patients with metastatic disease and previously unirradiated weight-bearing bones with pathological or impending pathological fracture, almost exclusively of the femur (5). A total of 35 sites that received adjuvant RT were compared to 29 sites that were treated with surgery alone. The authors stated that postoperative RT is the most important 
factor achieving and maintaining a normal functional status. In that study, postoperative RT was also associated with fewer orthopedic procedures as well as with an improved overall survival.

Wedin et al report in a retrospective study that the reoperation rate in irradiated fracture sites was not significantly different as compared with nonirradiated sites (13 versus 10 percent). In their study, 192 patients were treated surgically for 228 metastatic lesions of the long bones, 60 percent of the patients received preoperative or postoperative RT (14). However, in the study of Wedin et al., five of the six patients who required surgery for local tumor progression had not received RT. Our data failed to demonstrate a statistically significant reduction in the reoperation rate after radiotherapy, too, but it probably was due to the small number of patients, who did not received adjuvant RT. The incidence of local tumor progress was significantly lower in patients who were treated with RT, which is in line with the results of Wedin et al. Given the retrospective nature of our study and the potential for selection bias, outcomes must be interpreted cautiously. Nevertheless, we could demonstrate that dispense with radiotherapy after surgical stabilization of complete or impending pathologic increases the risk of pain flare and/or local tumor progress. A multidisciplinary approach and treatment, including surgical stabilization, radiotherapy and systemic treatment delivers the best results.

\section{Conclusions}

Our data confirm the efficacy and necessity of postoperative RT after orthopedic stabilization for metastatic bone disease to control the local disease. Bisphosphonates do not obviate the need for RT in the management of bone metastases after surgical stabilization. The combined treatment might lead to a better local tumor and pain control.

\section{Competing Interests}

The authors have declared that no competing interest exists.

\section{References}

1. Damron TA, Sim FH. Surgical treatment for metastatic disease of the pelvis and the proximal end of the femur. Instr Course Lect 2000; 49:461.

2. Damron TA, Bogart JA, Bilsky M. Evaluation and management of complete and impending pathologic fractures in patients with metastatic bone disease, multiple myeloma, and lymphoma. UpToDate, Accessed on July 25, 2015.

3. Ogilvie, C.M., Fox, E.J., Lackman, R.D. Current surgical management of bone metastases in the extremities and pelvis. Semin Oncol. 2008; 35: 118-28

4. Biermann, J.S., Holt, G.E., Lewis, V.O., Schwartz, H.S., Yaszemski, M.J. Metastatic Bone Disease: Diagnosis, Evaluation, and Treatment. J Bone Joint Surg Am. 2009; 91: 1518-1530

5. Townsend, P.W., Smalley, S.R., Cozad, S.C., Rosenthal, H.G., Hassanein, R.E. Role of postoperative radiation therapy after stabilization of fractures caused by metastatic disease. Int J Radiat Oncol Biol Phys. 1995; 31: 43-9

6. Frassica D.A. General principles of external beam radiation therapy for skeletal metastases. Clin Orthop Relat Res. 2003; 415 Suppl: 158-64
7. Chow E., Harris K., Fan G., Tsao M.N., Sze W.M. Palliative radiotherapy trials for bone metastases: A systematic review. J Clin Oncol 2007; 25: 1423-1436

8. Tubiana-hulin M, Beuzeboc P, Mauriac L, et al. Double-blinded controlled study comparing clodronate versus placebo in patients with breast cancer bone metastases. Bull Cancer 2001, 88:701-707.

9. Hershman DL, Mcmahon DJ, Crew KD, et al. Zoledronic acid prevents bone loss in premenopausal women undergoing adjuvant chemotherapy for earlystage breast cancer. J Clin Oncol 2008, 26:4739-4745.

10. He M, Fan W, Zhang X Adjuvant zoledronic acid therapy for patients with early stage breast cancer: an updated systematicreview and meta-analysis. J Hematol Oncol. 2013; 6(1):80. doi: 10.1186/1756-8722-6-80.

11. Koswig S, Budach V. Remineralization and pain relief in bone metastases after after different radiotherapy fractions (10 times 3 Gy vs. 1 time $8 \mathrm{~Gy}$ ). A prospective study. Strahlenther Onkol. 1999; 175:500-8 [Article in German]

12. Feyer PC, Steingraeber M. Radiotherapy of Bone Metastasis in Breast Cancer Patients - Current Approaches. Breast Care (Basel). 2012;7(2):108-112.

13. Kouloulias V1, Matsopoulos G, Kouvaris I, et al. Radiotherapy in conjunction with intravenous infusion of $180 \mathrm{mg}$ of disodium pamidronate in management of osteolytic metastases from breast cancer: clinical evaluation, biochemical markers, quality of life, and monitoring of recalcification using assessments of gray-level histogram in plain radiographs. Int J Radiat Oncol Biol Phys. 2003; 57(1):143-57.

14. Wedin R, Bauer HC, Wersäll P. Failures after operation for skeletal metastatic lesions of long bones. Clin Orthop Relat Res 1999;(358):128-39. 